\title{
A numerical method for studying impulsively generated convection from heated tubes
}

\author{
S. J. D. D’Alessio \\ Department of Applied Mathematics, University of Waterloo, Canada
}

\begin{abstract}
This study presents a numerical method for solving the two-dimensional unsteady problem of laminar free convection from a heated tube in an otherwise quiescent fluid. The governing Navier-Stokes and energy equations are formulated in terms of the streamfunction and vorticity. The numerical scheme is designed to handle a large range of Grashof numbers and to capture the physical behaviour inherent in the initial flow. To numerically solve the governing equations a spectral finitedifference method is proposed. The temperature and vorticity are advanced in time using an implicit scheme of Crank-Nicholson type. The streamfunction, on the other hand, is expanded in a truncated Fourier series. To determine the surface vorticity exact integral conditions are derived and incorporated into the numerical method. The numerical results have been verified against derived analytical solutions which are valid for small times and large Grashof numbers. The numerical and analytical results are found to be in good agreement.

Keywords: unsteady, laminar, viscous, incompressible, Boussinesq, spectral finitedifference scheme.
\end{abstract}

\section{Introduction}

Free convection from a horizontal two-dimensional body is a fundamental thermalfluid problem. It has received numerous numerical, experimental and theoretical studies over the years. This paper deals with the unsteady behaviour of laminar, two-dimensional flow caused by free convection from a heated elliptic cylinder emitting a constant surface heat flux into the surrounding fluid which is initially at rest. This problem is of interest for both theoretical and practical reasons since it has important applications in engineering such as heat transfer from heated tubes, hot wire anemometry, thermal pollution and even in the design of heat exchangers. 
The present work differs from previous studies (summarized in [1]) in several respects. First, the majority of the previous studies have focussed on circular cylinders which is much simpler. Second, a new numerical approach based on a different scaling of the equations is proposed. Third, an approximate analytical solution is provided and used to validate the numerical solution for small times and large Grashof numbers.

\section{Governing equations}

The equations governing the motion of a viscous incompressible fluid are the Navier-Stokes and energy equations. The fluid is characterized by properties which include: $\nu$ the kinematic viscosity, $\kappa$ the thermal diffusivity, $\alpha$ the thermal expansion coefficient, and $k$ the thermal conductivity. While these fluid properties are assumed to be constant, the fluid density, $\rho$, is allowed to vary with temperature, $T$, in the usual fashion

$$
\rho(T)=\rho_{0}\left[1-\alpha\left(T-T_{0}\right)\right]
$$

where $\rho_{0}$ refers to a reference density and $T_{0}$ a reference temperature. The flow setup is illustrated in Figure 1. To render the equations in dimensionless form the chosen length scale is the semi-focal length of the ellipse, $c=\sqrt{a^{2}-b^{2}}$, the time scale is $c / U$ where $U$ is the velocity scale (soon to be specified) and the temperature scale $\Delta T$ is related to the surface heat flux, $Q$, through $\Delta T=c Q / k$. The velocity scale is taken to be $U=(\alpha g \Delta T c)^{1 / 2}$ where $g$ is the acceleration due to gravity.

Since the flow is assumed to remain two-dimensional it is beneficial to work in terms of a streamfunction and vorticity. Also, because of the geometry of the problem it is worthwhile to work with the modified polar coordinates $(\xi, \theta)$ which are related to the Cartesian coordinates $(x, y)$ through the conformal transformation

$$
x+i y=\cosh \left[\left(\xi+\xi_{0}\right)+i \theta\right] .
$$

The advantage of this is that the contour of the cylinder is transformed to $\xi=0$ while the infinite region exterior to the cylinder is mapped to the semi-infinite rectangular strip $0<\xi<\infty,-\pi \leq \theta \leq \pi$. The constant $\xi_{0}$ is defined by

$$
\tanh \xi_{0}=r
$$

where $r=b / a$ is the ellipse aspect ratio equal to the ratio of the semi-minor to semi-major axis lengths. The above mapping holds for all elliptic cylinders having $0<r<1$ with $r=0$ denoting a flat plate and $r=1$ a circular cylinder. Another important feature associated with this transformation is that length scales close to the cylinder remain unchanged while those far away get contracted. This is helpful from a numerical point of view since the flow field is compressed. 


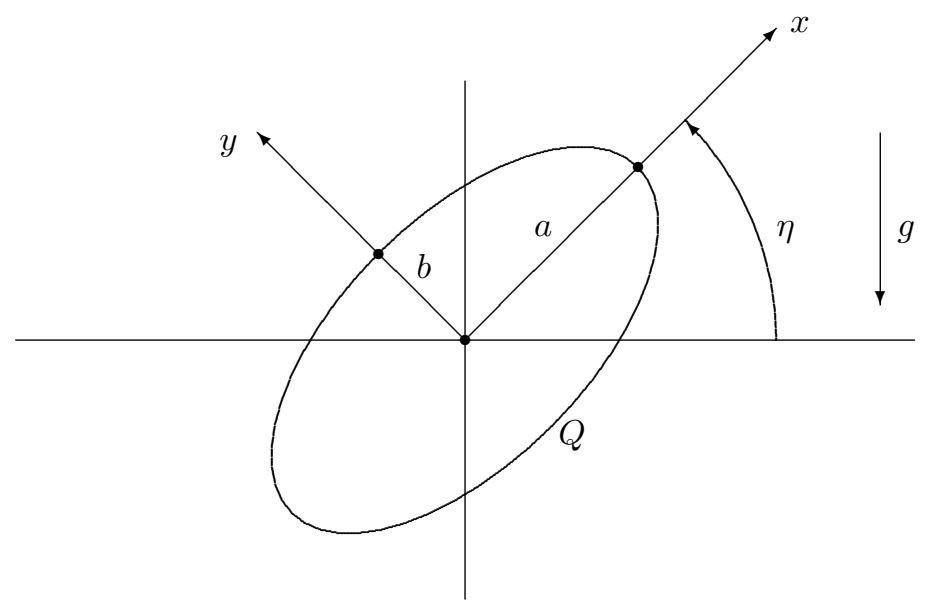

Figure 1: The flow setup.

In terms of the coordinates $(\xi, \theta)$ the dimensionless unsteady Navier-Stokes and energy equations for a viscous, incompressible fluid in terms of the streamfunction, $\psi$, vorticity, $\zeta$, and temperature, $\phi$, then become

$$
\begin{gathered}
\frac{\partial^{2} \psi}{\partial \xi^{2}}+\frac{\partial^{2} \psi}{\partial \theta^{2}}=M^{2} \zeta \\
\frac{\partial \zeta}{\partial t}=\frac{1}{M^{2}}\left[\frac{\partial \psi}{\partial \theta} \frac{\partial \zeta}{\partial \xi}-\frac{\partial \psi}{\partial \xi} \frac{\partial \zeta}{\partial \theta}+\frac{1}{\sqrt{G r}}\left(\frac{\partial^{2} \zeta}{\partial \xi^{2}}+\frac{\partial^{2} \zeta}{\partial \theta^{2}}\right)+A \frac{\partial \phi}{\partial \xi}-B \frac{\partial \phi}{\partial \theta}\right] \\
\frac{\partial \phi}{\partial t}=\frac{1}{M^{2}}\left[\frac{\partial \psi}{\partial \theta} \frac{\partial \phi}{\partial \xi}-\frac{\partial \psi}{\partial \xi} \frac{\partial \phi}{\partial \theta}+\frac{1}{\sqrt{G r} P r}\left(\frac{\partial^{2} \phi}{\partial \xi^{2}}+\frac{\partial^{2} \phi}{\partial \theta^{2}}\right)\right]
\end{gathered}
$$

where

$$
\begin{gathered}
M^{2}=\frac{1}{2}\left[\cosh \left(2\left(\xi+\xi_{0}\right)\right)-\cos (2 \theta)\right], \\
A=\sinh \left(\xi+\xi_{0}\right) \cos (\eta) \cos (\theta)-\cosh \left(\xi+\xi_{0}\right) \sin (\eta) \sin (\theta), \\
B=\cosh \left(\xi+\xi_{0}\right) \cos (\eta) \sin (\theta)+\sinh \left(\xi+\xi_{0}\right) \sin (\eta) \cos (\theta) .
\end{gathered}
$$

The problem as posed is completely specified by the following dimensionless parameters: the Grashof number, $G r=\alpha g c^{3} \Delta T / \nu^{2}$, the inclination, $\eta$, the Prandtl number, $\operatorname{Pr}=\nu / \kappa$, and the ellipse parameter, $r$. The dimensionless temperature, $\phi$, is related to the dimensional temperature, $T$, through $\phi=(T-$ $\left.T_{0}\right) / \Delta T$. Similarly, $\psi=\tilde{\psi} /(c U)$ and $\zeta=c \tilde{\zeta} / U$ with the tilde denoting a dimensional quantity. Lastly, in arriving at the above equations we have made the Boussinesq approximation to describe the buoyancy force and have omitted viscous dissipation.

We assume that at $t=0$ an impulsive heat flux is applied to the cylinder surface and that both the cylinder surface and surrounding fluid have an initial temperature 
of $T_{0}$. Equations (1)-(3) are to be solved subject to the no-slip and constant flux conditions on the surface given by

$$
\psi=\frac{\partial \psi}{\partial \xi}=0 \text { and } \frac{1}{M} \frac{\partial \phi}{\partial \xi}=-1 \quad \text { on } \quad \xi=0 .
$$

Inspecting these conditions we observe that two conditions for the streamfunction are given while none for the vorticity is provided. Later we will discuss a method to prescribe the surface vorticity. In [2], the vorticity field is shown to satisfy integral constraints. These can be derived from the no-slip boundary conditions using Green's second identity and are given by:

$$
\begin{aligned}
& \int_{0}^{\infty} \int_{-\pi}^{\pi} e^{-n \xi} M^{2} \zeta \sin (n \theta) d \theta d \xi=0, n=1,2, \cdots \\
& \int_{0}^{\infty} \int_{-\pi}^{\pi} e^{-n \xi} M^{2} \zeta \cos (n \theta) d \theta d \xi=0, n=0,1, \cdots
\end{aligned}
$$

At large distances we impose

$$
\psi, \zeta, \phi \rightarrow 0 \text { as } \xi \rightarrow \infty,
$$

which correspond to a quiescent far-field flow. Lastly, we need to specify initial conditions. Since the fluid initially has a uniform temperature and the motion starts from rest, the initial conditions are simply

$$
\psi(\xi, \theta, t=0)=\zeta(\xi, \theta, t=0)=\phi(\xi, \theta, t=0)=0 .
$$

To better resolve the early stages of the flow following the impulsive startup at $t=0$, the boundary-layer coordinate, $z$, defined by

$$
\xi=\lambda z, \lambda=\sqrt{\frac{4 t}{\sqrt{G r}}},
$$

is used. Essentially, this change of variable stretches the thermal-boundary layer. In terms of the coordinate $z$ equations (1)-(3) get transformed to

$$
\begin{gathered}
\frac{\partial^{2} \psi}{\partial z^{2}}+\lambda^{2} \frac{\partial^{2} \psi}{\partial \theta^{2}}=\lambda^{2} M^{2} \zeta \\
\frac{1}{M^{2}} \frac{\partial^{2} \zeta}{\partial z^{2}}+2 z \frac{\partial \zeta}{\partial z}=4 t \frac{\partial \zeta}{\partial t}-\frac{\lambda^{2}}{M^{2}} \frac{\partial^{2} \zeta}{\partial \theta^{2}} \\
+\frac{4 t}{\lambda M^{2}}\left(\frac{\partial \psi}{\partial z} \frac{\partial \zeta}{\partial \theta}-\frac{\partial \psi}{\partial \theta} \frac{\partial \zeta}{\partial z}\right)-\frac{4 t A}{\lambda M^{2}} \frac{\partial \phi}{\partial z}+\frac{4 t B}{M^{2}} \frac{\partial \phi}{\partial \theta} \\
\frac{1}{\operatorname{Pr} M^{2}} \frac{\partial^{2} \phi}{\partial z^{2}}+2 z \frac{\partial \phi}{\partial z}=4 t \frac{\partial \phi}{\partial t}-\frac{\lambda^{2}}{\operatorname{Pr} M^{2}} \frac{\partial^{2} \phi}{\partial \theta^{2}}+\frac{4 t}{\lambda M^{2}}\left(\frac{\partial \psi}{\partial z} \frac{\partial \phi}{\partial \theta}-\frac{\partial \psi}{\partial \theta} \frac{\partial \phi}{\partial z}\right)
\end{gathered}
$$

As a final note we emphasize that although the boundary-layer coordinate $z$ is utilized, the fully nonlinear Navier-Stokes and energy equations are to be solved and not the simplified boundary-layer equations. 


\section{Numerical solution procedure}

As previously mentioned the early stages of the flow are to be computed using equations (5)-(7) involving the boundary-layer coordinate $z$. Once the boundary layer thickens appreciably one can switch back to the original coordinate $\xi$ and solve equations (1)-(3). However, for large $G r$ it is more practical to work entirely in the boundary-layer coordinate $z$. In the numerical scheme outlined below only the procedure for solving equations (5)-(7) will be discussed for the sake of brevity. The procedure for solving (1)-(3) will be very similar.

We begin by discretizing the computational domain bounded by $0 \leq z \leq z_{\infty}$ and $-\pi \leq \theta \leq \pi$ into a uniform network of $K \times L$ grid points located at

$$
\begin{gathered}
z_{i}=i h_{z}, i=0,1, \ldots, K \text { where } h_{z}=\frac{z_{\infty}}{K} \\
\text { and } \theta_{j}=-\pi+j h_{\theta}, j=0,1, \ldots, L \text { where } h_{\theta}=\frac{2 \pi}{L},
\end{gathered}
$$

with $z_{\infty}$ denoting the outer boundary approximating infinity.

The streamfunction is solved by expanding it into a truncated Fourier series

$$
\psi(z, \theta, t)=\frac{1}{2} F_{0}(z, t)+\sum_{n=1}^{N}\left[F_{n}(z, t) \cos (n \theta)+f_{n}(z, t) \sin (n \theta)\right] .
$$

where the Fourier coefficients satisfy

$$
\begin{gathered}
\frac{\partial^{2} F_{n}}{\partial z^{2}}-n^{2} \lambda^{2} F_{n}=\lambda^{2} s_{n}(z, t), n=0,1, \cdots \\
\frac{\partial^{2} f_{n}}{\partial z^{2}}-n^{2} \lambda^{2} f_{n}=\lambda^{2} r_{n}(z, t) ;, n=1, \cdots
\end{gathered}
$$

with

$$
\begin{aligned}
& s_{n}(z, t)=\frac{1}{\pi} \int_{-\pi}^{\pi} M^{2} \zeta \cos (n \theta) d \theta, \\
& r_{n}(z, t)=\frac{1}{\pi} \int_{-\pi}^{\pi} M^{2} \zeta \sin (n \theta) d \theta .
\end{aligned}
$$

Boundary conditions for the Fourier components can easily be determined from those for the streamfunction. Further conditions satisfied by the functions $r_{n}(z, t)$ and $s_{n}(z, t)$ follow from the integral conditions and are given by

$$
\begin{gathered}
\int_{0}^{\infty} \mathrm{e}^{-n \lambda z} s_{n}(z, t) d z=0, n=0,1,2, \cdots \\
\int_{0}^{\infty} \mathrm{e}^{-n \lambda z} r_{n}(z, t) d z=0, n=1,2, \cdots .
\end{gathered}
$$

The above play an important role in determining the surface vorticity. 
Equations (8)-(9) at a fixed time are of the form

$$
h^{\prime \prime}(z)-\beta^{2} h(z)=g(z),
$$

where $\beta=n \lambda$ and the prime refers to differentiation with respect to $z$. These ordinary differential equations can be integrated using step-by-step formulae. The important point to note here is that the particular marching algorithm to be used is dependent on the parameter $\beta$. Two sets of step-by-step methods were utilized: one for $\beta<0.5$ while another one for $\beta \geq 0.5$. The specific schemes used can be found in [3].

To discuss the numerical method used to solve equations (6)-(7) we begin by rewriting them in the generic form

$$
t \frac{\partial \chi}{\partial t}=q(z, \theta, t)
$$

The scheme used to discretize this equation is very similar to the Crank-Nicholson implicit procedure. Assuming the solution at time $t$ is known, we advance the solution to time $t+\Delta t$ by integrating the above. Integration by parts yields

$$
\left.\chi \tau\right|_{t} ^{t+\Delta t}-\int_{t}^{t+\Delta t} \chi d \tau=\int_{t}^{t+\Delta t} q d \tau
$$

where $\Delta t$ is the time increment. Approximating the integrals using the trapezoidal rule results in the expression

$$
\chi(z, \theta, t+\Delta t)=\chi(z, \theta, t)+\left(\frac{\Delta t}{2 t+\Delta t}\right)[q(z, \theta, t+\Delta t)+q(z, \theta, t)] .
$$

Since $q(z, \theta, t+\Delta t)$ depends on $\chi(z, \theta, t+\Delta t)$ and its spacial derivatives the scheme is implicit. This equation is solved iteratively using a Gauss-Seidel procedure. All spatial derivatives appearing in the function $q$ are approximated using central-differences; thus the scheme given is second order accurate in both space and time.

The boundary conditions used in solving the energy equation are straightforward and require no explanation. For the vorticity transport equation, on the other hand, careful attention must be given to determine the surface vorticity. The surface vorticity can be determined by inverting the expressions for $r_{n}$ and $s_{n}$ and leads to the truncated Fourier series

$$
\zeta(0, \theta, t)=\frac{1}{M_{0}^{2}}\left\{\frac{1}{2} s_{0}(0, t)+\sum_{n=1}^{N}\left[r_{n}(0, t) \sin (n \theta)+s_{n}(0, t) \cos (n \theta)\right]\right\},
$$

where $M_{0}^{2}=M^{2}(z=0, \theta)$. The quantities $s_{n}(0, t)$ and $r_{n}(0, t)$ are computed by enforcing the integral conditions; that is, off the cylinder surface $r_{n}$ and $s_{n}$ can be computed using the most recent guess for $\zeta$. Then, $s_{n}(0, t)$ and $r_{n}(0, t)$ are computed by numerically satisfying the integral constraints. 
We summarize the numerical method by listing the steps involved in the procedure. Assuming all quantities are known at time $t$ and wish to advance the solution to a time $t+\Delta t$, we perform the following steps ( $p$ denotes the iteration counter ):

1. solve for $\phi^{(p)}(z, \theta, t+\Delta t)$,

2. solve for $\zeta^{(p)}(z, \theta, t+\Delta t)$ everywhere except on the cylinder surface $(z=0)$,

3. compute $r_{n}^{(p)}(z, t+\Delta t), s_{n}^{(p)}(z, t+\Delta t)$ for $z \neq 0$,

4. calculate $r_{n}^{(p)}(0, t+\Delta t), s_{n}^{(p)}(0, t+\Delta t)$ by enforcing the integral conditions and hence compute $\zeta^{(p)}(0, \theta, t+\Delta t)$,

5. solve for $f_{n}^{(p)}(z, t+\Delta t), F_{n}^{(p)}(z, t+\Delta t)$ and thus obtain $\psi^{(p)}(z, \theta, t+\Delta t)$,

6. repeat above steps till convergence is reached and increment $p$ by 1 after each complete iteration.

Step (4) indicates how the integral conditions are used in determining the surface vorticity. It may also be necessary to subject the surface vorticity to underrelaxation in order to obtain convergence. Convergence is reached when the difference between two successive iterates of the surface vorticity falls below some specified tolerance $\epsilon$.

\section{Results and discussion}

After performing numerous numerical experiments, the following computational parameters were chosen: $N=25, \epsilon=10^{-6}, z_{\infty}=10$. A typical grid size used was $K \times L=200 \times 120$. Because of the impulsive start, small time steps of $\Delta t=10^{-3}$ were used initially. As time increased the time step was gradually increased to $\Delta t=0.05$. Results were obtained for parameter values of $r=0.5$, $\eta=45^{\circ}, \operatorname{Pr}=0.7$ (corresponding to air) with $G r=10^{2}$ and $G r=10^{4}$.

Shown in Figures 2(a), (b) are isotherm plots at times $t=2.5,100$, respectively, for the case $G r=10^{2}$. In all isotherm plots to be presented the outermost contour corresponds to $\phi=0.05$ and the spacing between consecutive contours was set to $\Delta \phi=0.05$. For this case computations were carried out in the boundary-layer coordinate up to $t=2.5$ and then switched back to the original coordinate. At $t=2.5$ the parameter $\lambda=1$ and thus is a convenient time to switch coordinates. The isotherms portrayed in Figure 2(a) appear to form concentric rings. For small times this is to expected as this corresponds to the conduction regime. For large times, as depicted in Figure 2(b), a well developed thermal plume forms.

Next we present some results for the case $G r=10^{4}$. For this large Grashof number computations were carried out entirely in the boundary-layer coordinate. Displayed in Figure 3 is an isotherm plot at $t=20$. Witnessed in this diagram is the formation of a well defined plume. It is interesting to note that for large $G r$ the plume develops much earlier in time due to the enhanced buoyancy force.

Surface temperature and vorticity distributions are plotted in Figures 4, 5, respectively, at various times for the case $G r=10^{4}$. As time advances both distributions reveal a prominent maximum evolving. 
432 Computational Methods and Experimental Measurements XIII

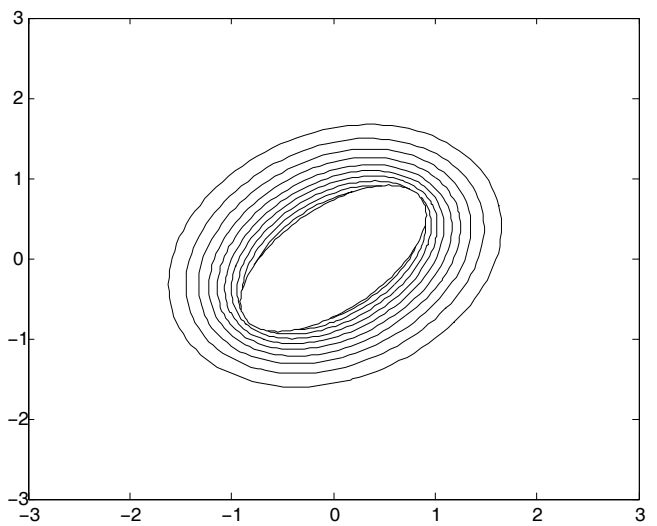

(a)

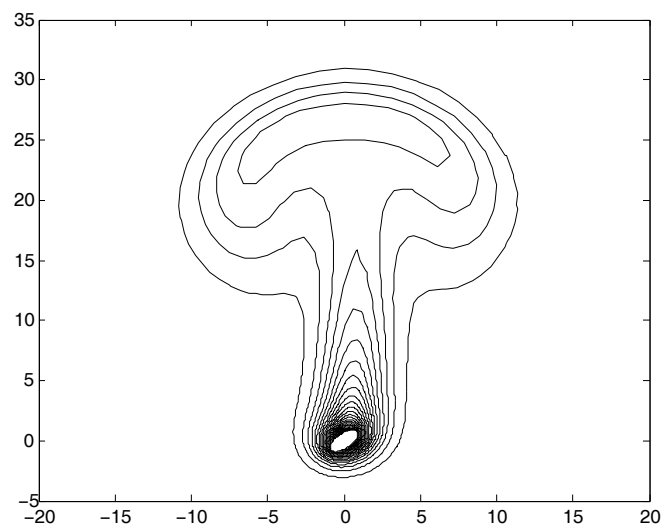

(b)

Figure 2: (a) Isotherms at $t=2.5$ for $G r=10^{2}$, (b) isotherms at $t=100$ for $G r=10^{2}$.

The numerical scheme was verified against a derived analytical solution which is valid for small $t$ and large $G r$. If $G r$ is large and $t$ is small, then $\lambda$ is also small, and it is possible to expand the flow variables in a double series in terms of $\lambda$ and $t$. First, each flow variable $\chi$ is expanded in a series of the form

$$
\chi=\chi_{0}+\lambda \chi_{1}+\lambda^{2} \chi_{2}+\cdots
$$

Then each $\chi_{n}, n=0,1,2, \cdots$, is further expanded in a series of the form

$$
\chi_{n}(z, \theta, t)=\chi_{n 0}(z, \theta)+t \chi_{n 1}(z, \theta)+\cdots .
$$


Computational Methods and Experimental Measurements XIII 433

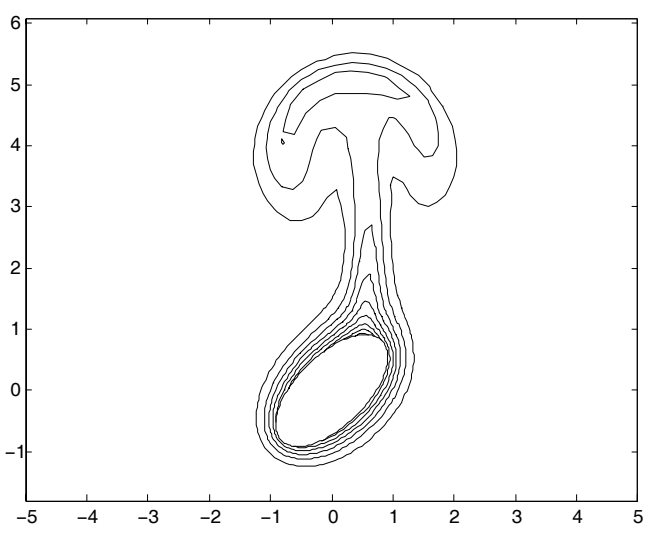

Figure 3: Isotherms at $t=20$ for $G r=10^{4}$.

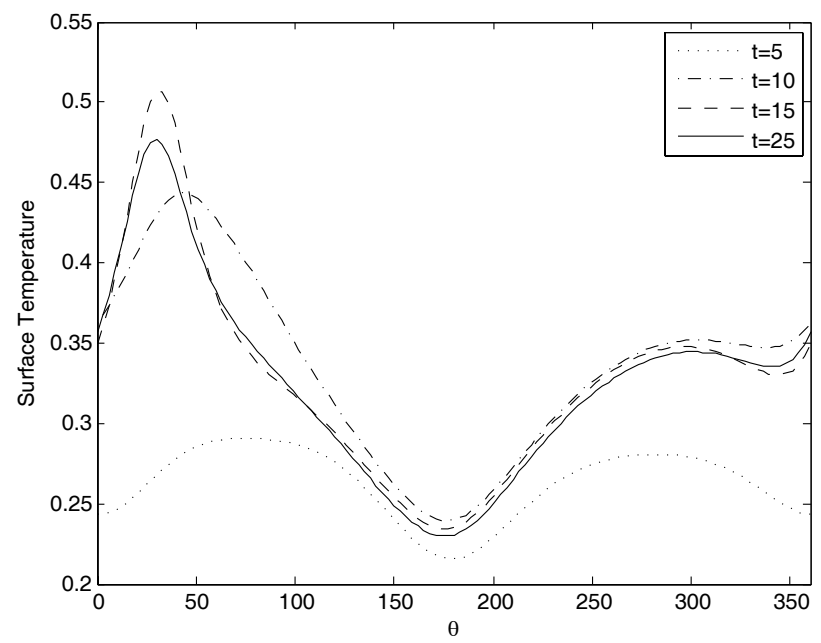

Figure 4: Surface temperature distributions at various times for $G r=10^{4}$.

Following this procedure, the leading-order term in the solution for the temperature can be shown to be

$$
\phi(z, \theta, t) \sim \frac{2 \sqrt{t}}{\sqrt{\pi P r \sqrt{G r}}}\left(\mathrm{e}^{-\operatorname{Pr} M_{0}^{2} z^{2}}-\sqrt{\pi \operatorname{Pr}} M_{0} z \operatorname{erfc}\left(\sqrt{\operatorname{Pr}} M_{0} z\right)\right)
$$

$$
\text { where } \operatorname{erfc}(\mathbf{x})=1-\frac{2}{\sqrt{\pi}} \int_{0}^{x} \mathrm{e}^{-w^{2}} d w .
$$


434 Computational Methods and Experimental Measurements XIII

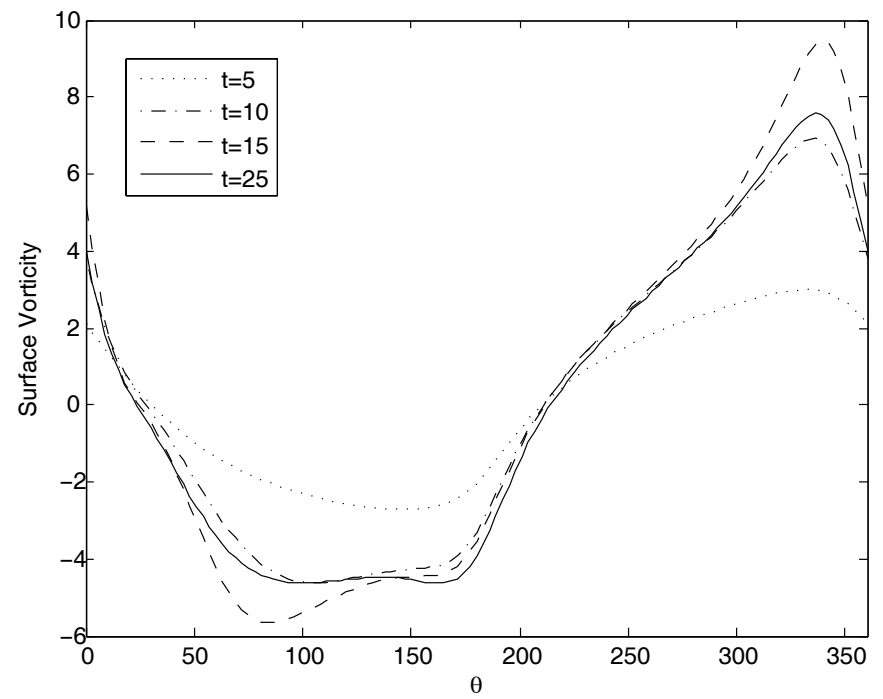

Figure 5: Surface vorticity distributions at various times for $\mathrm{Gr}=10^{4}$.

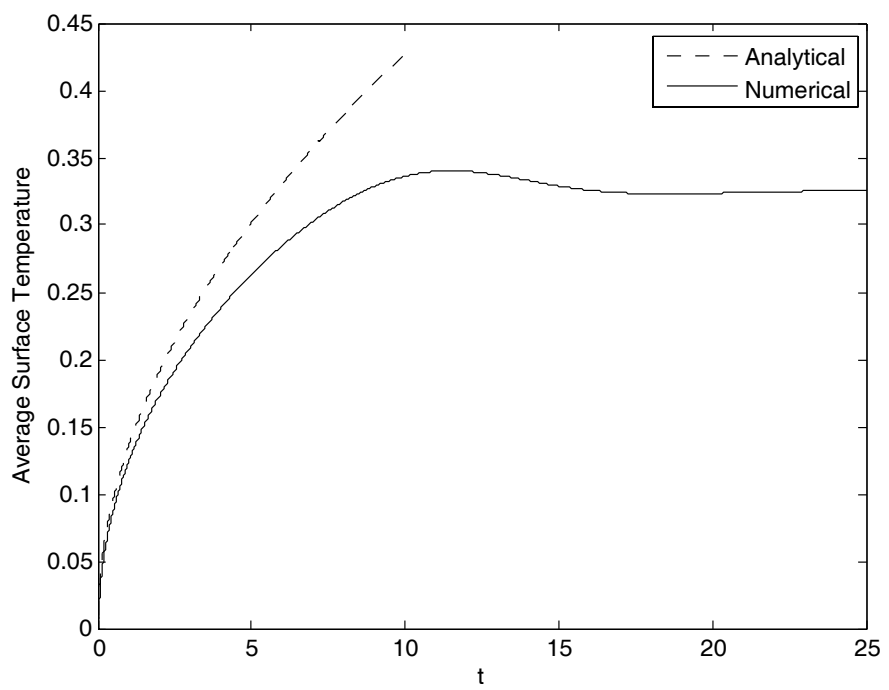

Figure 6: Comparison between analytical and numerical solutions for $G r=10^{4}$.

Contrasted in Figure 6 are the analytical and numerical solutions for $G r=10^{4}$. As expected, for small $t$ the two solutions are in good agreement and as time progresses the agreement worsens. 


\section{Concluding remarks}

Discussed in this paper is a numerical procedure to solve the problem of impulsively generated free convection from a heated tube. Although the details were only presented for the case of an elliptic cylinder, other cylinder cross sections can easily be handled by simply changing the metric, $M^{2}$, in equations (1)-(3). A numerical technique involving both finite difference and spectral methods was described and was successful in computing the unsteady flow for a large range of Grashof numbers.

Future work involves continuing the analytical solution procedure and determining more terms in the expansion. In addition, it is desirable to make connections with the recent experimental results of Elsayed et al. [4] to demonstrate that the proposed numerical method can realistically mimic the physical problem in the laminar regime.

\section{Acknowledgement}

Financial support for this research was provided by the Natural Sciences and Engineering Research Council of Canada.

\section{References}

[1] Saitoh, T., Sajiki, T.\& Maruhara, K., Bench mark solutions to natural convection heat transfer problem around a horizontal circular cylinder, International Journal of Heat and Mass Transfer 36, pp. 1251-1259, 1993.

[2] Dennis, S.C.R. \& Quartapelle, L., Some uses of Green's theorem in solving the Navier-Stokes equations, International Journal of Numerical Methods in Fluids 9, pp. 871-890, 1989.

[3] Staniforth, A.N., Studies of symmetrical and asymmetrical viscous flows past impulsively started cylinders, Ph.D. thesis, University of Western Ontario, London, Canada, 1972.

[4] Elsayed, A.O., Ibrahim, E.Z.\& Elsayed, S.A., Free convection from a constant heat flux elliptic tube, Energy Conversion and Management 44, pp. 24452453, 2003. 